\title{
Hemodynamic Features of Non-Aneurysmal Subarachnoid Hemorrhage in a Case of Familial Moyamoya Disease: A Transcranial Doppler Ultrasound Study
}

\author{
Massimiliano Toscano Francesca Puledda Alessandro Viganò Edoardo Vicenzini \\ Giulio Guidetti Gian Luigi Lenzi Vittorio Di Piero
}

Department of Neurology and Psychiatry, 'Sapienza' University of Rome, Rome, Italy

\section{Key Words \\ Moyamoya disease $\cdot$ Subarachnoid hemorrhage - Collateral circulation . Transcranial Doppler ultrasonography}

A very rare clinical manifestation of Moyamoya Disease (MMD) is subarachnoid hemorrhage, which is not due to the ruptured intracranial aneurysms (NASAH). This is an extremely rare condition and to our knowledge only five other cases [1-5] have been described in the literature; out of them only one was familial [3]. Moreover, there is currently no study reporting the hemodynamic features of intra and extracranial circulation assessed with ultrasound examination. We present data from a oneyear Transcranial Doppler ultrasound (TCD) follow up, in the case of a 57-yearold woman admitted to our hospital for a sudden onset of headache followed by a partial seizure. Family history revealed that two of her cousins had been diagnosed with MMD, one of which deceased at the age of 52 for a subarachnoid hemorrhage (SAH) of unknown cause. On admission, a CT scan revealed SAH over the right frontal cortex and parieto-occipital cortex bilaterally. Digital Subtraction Angiography showed a bilateral stenosis of the supraclinoid segment of the ICAs (for artery acronyms see table 1 legend), and an anomalous
Willis circle with absence of the ACAs and of L-MCA and multiple tortuous vessels branching from the R-MCA ('moyamoya vessels'). No aneurysms or vascular malformations were found (fig. 1).

Based on these findings, the patient was diagnosed with MMD. No neurological deficits were noted on the patient's physical examination. Upon admission, we performed a Carotid Duplex ultrasound and a TCD, which showed an increase in mean blood flow velocity of R-MCA. This was initially attributed to vasospasm and nimodipine therapy was started.

We also performed a progressive TCD follow-up; hemodynamic parameters of the first ultrasound examination as well as those from TCD follow-up are summarized in table 1 (we exclusively reported follow-up values from the R-MCA, which were the only ones that showed significant changes through time). The patient had a very favorable outcome and is currently asymptomatic.

Particularly interesting is the monitoring of hemodynamic state of brain vessels done in our case. According to previous studies performed on asymptomatic MMD patients [6], we found at T0 that both the ICAs showed normal flow velocity and low resistance, while the remaining intracranial vessels showed a consistent hemodynamic pattern in terms of high flow velocity and low resistance. This pattern re- mained stable in the follow up, suggesting that it might be representative of the collateral network typical of MMD. Interestingly, R-MCA showed a different behaviour in respect to the other vessels, with a very high flow velocity and normal flowresistive indices. In the acute phase, we attributed this pattern to initial vasospasm. However, no clinical or radiological signs of vasospasm were observed. Nonetheless, subsequent controls revealed a paradoxical increase in blood flow velocity along with the progressive lowering of resistance indices of the R-MCA.

Concerns exist regarding the predictive capacities of evaluating vasospasm by measuring the sole flow velocities: in literature, a good correlation between elevated flow velocity as shown by TCD and angiographically proven arterial narrowing has been widely accepted, as a matter of fact, only in those vessels with a minimal influence of collateralization [7]. This is probably not the case of our patient, given the presence of the abundant collateral vessels branching from the R-MCA. Steinmeier and colleagues [8] also found that the presence of SAH significantly increases pulsatility indices probably reflecting a global change in vascular peripheral resistance. Moreover, a negative correlation between increasing flow velocity and decreasing flow resistance has been found in arteries with constant di-

\section{KARGER}

E-Mail karger@karger.com

www.karger.com/ene (c) 2014 S. Karger AG, Base

0014-3022/14/0726-0330\$39.50/0
Massimiliano Toscano, MD

Department of Neurology and Psychiatry

'Sapienza' University of Rome

Viale dell'Università 30, 00185 Rome (Italy)

E-Mail massimiliano.toscano@uniromal.it 
Fig. 1. Right (R) and Left (L) cerebral angiogram showed bilateral stenosis of the internal carotid arteries, absence of the anterior cerebral arteries and left middle cerebral artery, and excluded the presence of intracranial aneurysms. R: Multiple moyamoya vessels branching from the right middle cerebral artery can be seen. L: The left side of the brain received anastomosis from the left posterior cerebral artery and external carotid artery, specifically the superficial temporal artery (STA).
$\mathbf{R}$
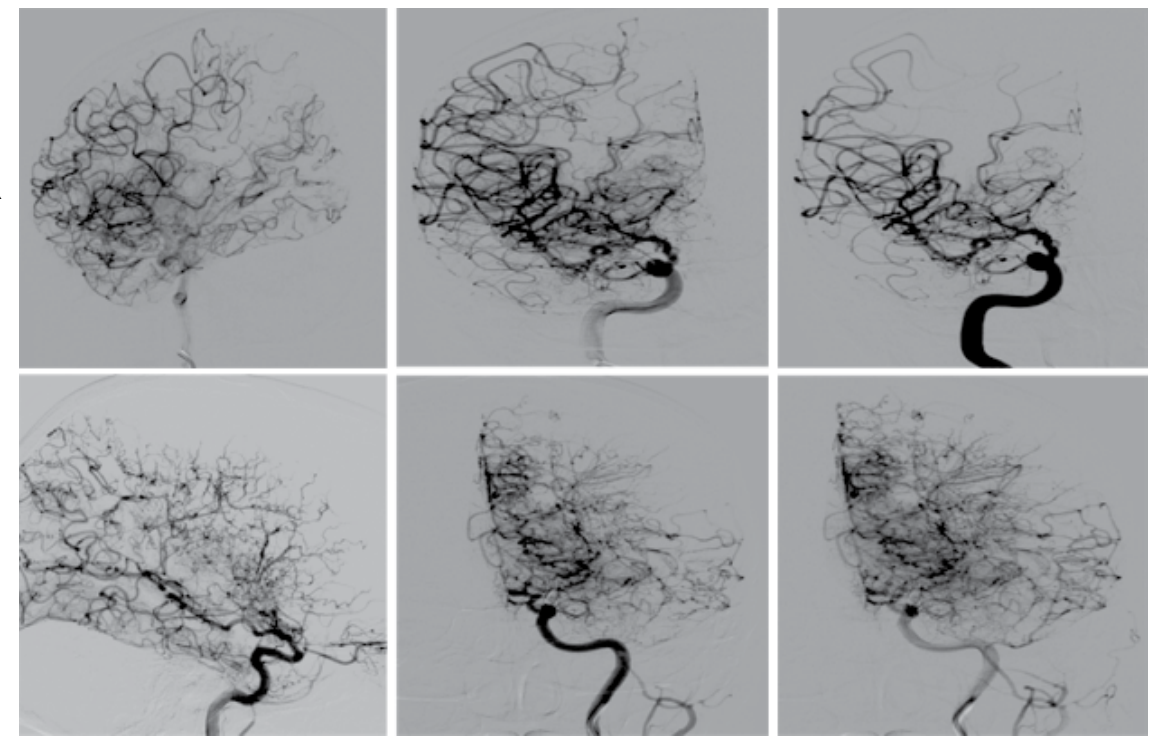

Table 1. Carotid Duplex ultrasound and Transcranial Doppler upon admission and follow up: hemodynamic parameters

\begin{tabular}{|c|c|c|c|c|c|c|}
\hline & $\mathrm{PSV}, \mathrm{cm} / \mathrm{s}$ & $\mathrm{EDV}, \mathrm{cm} / \mathrm{s}$ & $\mathrm{Vm}, \mathrm{cm} / \mathrm{s}$ & S/D & PI & RI \\
\hline \multicolumn{7}{|l|}{ Admission } \\
\hline L-ICA & 46.00 & 31.00 & 36.00 & 1.48 & 0.42 & 0.33 \\
\hline L-MCA & \multirow{2}{*}{\multicolumn{6}{|c|}{$\begin{array}{l}\text { not detected } \\
\text { not detected }\end{array}$}} \\
\hline L-ACA & & & & & & \\
\hline L-PCA & 77.00 & 54.00 & 61.67 & 1.43 & 0.37 & 0.30 \\
\hline $\mathrm{BA}$ & 76.00 & 54.00 & 61.33 & 1.41 & 0.36 & 0.29 \\
\hline R-ICA & 53.00 & 31.00 & 38.33 & 1.71 & 0.57 & 0.42 \\
\hline R-MCA & 188.00 & 77.00 & 114.00 & 2.44 & 0.97 & 0.59 \\
\hline R-ACA & \multicolumn{6}{|c|}{ not detected } \\
\hline R-PCA & 62.00 & 48.00 & 52.67 & 1.29 & 0.27 & 0.23 \\
\hline \multicolumn{7}{|c|}{ R-MCA follow-up } \\
\hline 2nd day & 178.00 & 79.00 & 112.00 & 2.25 & 0.88 & 0.56 \\
\hline 6 th day & 183.00 & 85.00 & 117.67 & 2.15 & 0.83 & 0.54 \\
\hline 9th day & 197.00 & 89.00 & 125.00 & 2.21 & 0.86 & 0.55 \\
\hline 22nd day & 201.00 & 108.00 & 139.00 & 1.86 & 0.67 & 0.46 \\
\hline 6 months & 212.00 & 113.00 & 147.33 & 1.84 & 0.66 & 0.46 \\
\hline 1 year & 209.00 & 113.00 & 145.00 & 1.85 & 0.66 & 0.46 \\
\hline
\end{tabular}

PSV = Peak systolic velocity; EDV = end-diastolic velocity; Vm = time-averaged mean flow velocity; $\mathrm{S} / \mathrm{D}=$ peak systolic velocity/ end-diastolic velocity ratio; $\mathrm{PI}=$ pulsatility index; $\mathrm{RI}=$ resistance index; $\mathrm{L}=$ left; $\mathrm{R}=$ right; $\mathrm{ICA}=$ internal carotid artery; $\mathrm{MCA}=$ middle cerebral artery; ACA = anterior cerebral artery; PCA = posterior cerebral artery; BA = basilar artery.

ameter [9]; therefore it could also be speculated that the progressive paradoxical increase in blood flow velocity in our patient (along with the progressive lowering of resistance indices), could actually represent a post-SAH reversion to a previous abnormal pattern typical of moyamoya collaterals (i.e., high flow velocity and low resistance) [6], more than vasospasm due to SAH.

These considerations could be of particular interest also from a clinical point of view: on the one hand, they highlight the role of TCD in over-time monitoring of hemodynamic state of brain vessels in MMD; on the other hand, they suggest that a peculiar hemodynamic pattern should probably be taken into account when considering nimodipine therapy in Moyamoya patients presenting with NASAH and suspected vasospasm, which therefore constitutes a challenging diagnostic issue. 


\section{References}

1 Alcala-Cerra GA, Moscote-Salazar LR, Barrios RS, et al: Non-aneurysmal subarachnoid hemorrhage as presentation of moyamoya disease in an adult. Surg Neurol Int 2011;2:80.

$\checkmark 2$ Fujimura M, Mugikura S, Shimizu H, et al: Asymptomatic moyamoya disease subsequently manifesting as transient ischemic attack, intracerebral hemorrhage, and subarachnoid hemorrhage in a short period: case report. Neurol Med Chir (Tokyo) 2010;50: 316.

>3 Matsumoto Y, Asada M, Mukubou M: Postpartum subarachnoid hemorrhage due to Moyamoya disease associated with renal artery stenosis. J Obstet Gynaecol Res 2009;35: 787.
4 Osanai T, Kuroda S, Nakayama N, et al: Moyamoya disease presenting with subarachnoid hemorrhage localized over the frontal cortex: case report. Surg Neurol 2008;69:197.

5 Marushima A, Yanaka K, Matsuki T, et al: Subarachnoid hemorrhage not due to ruptured aneurysm in moyamoya disease. J Clin Neurosci 2006;13:146.

-6 Lee YS, Jung KH, Roh JK: Diagnosis of moyamoya disease with transcranial Doppler sonography: correlation study with magnetic resonance angiography. J Neuroimaging 2004; 14:319.
7 Laumer R, Steinmeier R, Gönner F, et al: Cerebral hemodynamics in subarachnoid hemorrhage evaluated by transcranial Doppler sonography. Part 1. Reliability of flow velocities in clinical management. Neurosurgery 1993; 33:1.

8 Steinmeier R, Laumer R, Bondár I, et al: Cerebral hemodynamics in subarachnoid hemorrhage evaluated by transcranial Doppler sonography. Part 2. Pulsatility indices: normal reference values and characteristics in subarachnoid hemorrhage. Neurosurgery 1993; 33:10.

9 Giller CA, Hodges K, Batjer HH: Transcranial Doppler pulsatility in vasodilation and stenosis. J Neurosurg 1990;72:901. 